\title{
Ferromagnetism in graphene traced to an antisymmetric orbital combination of involved electronic states
}

\author{
Wei $\mathrm{Xu}$ and J. G. Che* \\ Surface Physics Laboratory (National Key Laboratory), \\ Key Laboratory of Computational Physical Sciences (MOE), \\ Department of Physics and Collaborative Innovation Center of Advanced Microstructures, \\ Fudan University, Shanghai 200433, People's Republic of China
}

\begin{abstract}
Based on first principles calculations, we reveal that the origin of ferromagnetism caused by $s p$ electrons in graphene with vacancies can be traced to electrons partially filling $s p^{2 *}$-antibonding and $p_{z}^{*}$-nonbonding states, which are induced by the vacancies and appear near the Fermi level. Because the spatial wavefunctions of the both states are composed of atomic orbitals in an antisymmetric configuration, their spin wavefunctions should be symmetric according to the electron exchange antisymmetric principle, leading to electrons partially filling these states in spin polarization. Since this $p_{z}^{*}$ state originates not from interactions between the atoms but from the unpaired $p_{z}$ orbitals due to the removal of $p_{z}$ orbitals on the minority sublattice, the $p_{z}^{*}$ state is constrained, distributed on the atoms of the majority sublattice, and decays gradually from the vacancy as $\sim 1 / r$. According to these characteristics, we concluded that the $p_{z}^{*}$ state plays a critical role in magnetic ordering in graphene with vacancies. If the vacancy concentration in graphene is large enough to cause the decay-length regions to overlap, constraining the $p_{z}^{*}$ orbital components as little as possible on the minority sublattice atoms in the overlap regions results in the vacancy-induced $p_{z}^{*}$ states being coherent. The coherent process in the overlap region leads to the wavefunctions in all the involved regions antisymmetrized, consequently causing ferromagnetism according to the electron exchange antisymmetric principle. This unusual mechanism concerned with the origin of $s p$-electron magnetism and magnetic ordering has never before been reported and is distinctly different from conventional mechanisms. Consequently, we can explain how such a weak magnetization with such a high critical temperature can be experimentally observed in proton-irradiated graphene.
\end{abstract}

PACS numbers: 75.50.Dd,75.70.Ak,73.22.Pr,75.75-c

\section{INTRODUCTION}

The present work examines the origin of magnetism in nominally nonmagnetic materials with only $s p$-electrons, which is the one of the most controversial issues in modern materials science ${ }^{1 / 5}$, by considering graphene containing vacancies as an example. Magnetism induced by removing a single $p_{z}$ orbital from the $\pi$-electron systems, such as graphene or graphite, have been observed by experiments at room temperature and have been predicted by calculations $\sqrt[36]{6}$. However, it is still quite controversial. Nair et al. reported that no magnetic ordering could be detected down to liquid helium temperatures 9 ? This phenomenon has attracted a significant amount of attention owing to its potential applications in spintronics, nanostructures and biocompatible materials. Before they can be regarded as candidate materials for the applications, a comprehensive understanding of the origin and coupling of the magnetic moments $(\mathrm{MM})$ in these materials is required ${ }^{14}$. However, the basic theory concerning magnetism in solids established by Heisenberg in the 1920's $\$$ stressed that to cause magnetism in solids, the principal quantum number of the electrons must be greater than or equal to three $(n \geq 3)$. Therefore, 40 years ago, when observations of magnetism in light-element materials (containing only $s p$ electrons, $n<3$ ) were reported, the first response was that these samples might be contaminated by mag- netic impurities 11 . In recent years, with the reports of observed magnetism in such materials having continuously increased and carefully analyses having excluded impurities as their magnetic origin $13|12| 13$, the evidence for ferromagnetic properties in these nominally nonmagnetic materials is considered firm. However, the magnetic origin or the mechanism responsible for the magnetism in these nominally nonmagnetic materials remains rather unclear $1|2| 4|8| 14,16$.

Even though it was still debated for FM or PM in irradiated graphene, we would focus on the serious difficulties met by theoretical study in the following two fronts: first, the origin of magnetism in $s p$ electron materials $(n<3)$, and second, how such small magnetization (three or four orders of magnitude smaller than conventional magnets) and such long-range magnetic coupling (a distance of $20 \AA$ between vacancies) can be ferromagnetic at room temperature 8 17 19 .

On the first front, the current explanation is based on Hund's rule 27 because the states to which magnetism can be traced are induced by vacancies, which help localize $s p$ electrons, such as those in isolated atoms. If this magnetism is observed in experiments, it could be understood using Hund's rule because atom-like-localized electrons could actually exist in materials. However, if this magnetism emerges from calculations based on band theory and on the singlet-electron approximation (SEA), one needs to be careful with the interpretation. Within the framework of band theory, the eigenstates are Bloch 
modes that are all extended according to the Bloch theorem 28 . As such, the magnetism arising from calculations based on band theory cannot be attributed to the localized nature of electrons per se because localized electrons do not exist in calculations based on SEA and the Bloch theorem. Even if the dispersion of a band looks flat, the electrons on this band are still extended, moving everywhere in crystale 28 .

On the second front, if Heisenberg's model is used to explain magnetic ordering in such materials, the contradictions exist for weak magnetization (three or four orders of magnitude smaller than that in classic magnets such iron), long-range magnetic orders (that is, a low vacancy concentration $\underline{3|13| 29}$ ), and high critical temperature (higher than room temperature 3 17 19]. Note that other models for this subject, such as the superexchange model, the double exchange model and the RKKY model, can essentially be seen as extensions of the Heisenberg model, because they all have a term of a scalar product of total spin moments on atoms $i$ and $j, \mathbf{S}_{i} \cdot \mathbf{S}_{j}$. The differences between these models lie only in the coupling method.

Clearly, there must be more substantial physics behind the magnetism in nominally nonmagnetic materials (without $d$ or $f$ electrons) and there must be an unusual mechanism different from the conventional mechanism in $d f$-electron solids. This is a great challenge and requires a root-and-branch rework of magnetic theory 6 .

In the present study, we report that the magnetism in graphene with vacancies can be traced to electrons partially occupying $s p^{2 *}$-antibonding states or $p_{z}^{*}$ nonbonding states because the spatial wavefunctions of the both states are antisymmetric and their spin wavefunctions should be symmetric according to the electron exchange antisymmetric principle. This is the origin of the magnetism induced by $s p$ electrons. It has been recognized that the nonbonding state (or zero mode in literature 22130 $)$ decays with $\sim 1 / r$ ( $r$ being the distance to the vacancy) with long-range interaction 2230 . However, it is still unclear how the long-range interaction between these states created by vacancies can be so strong to couple such weak magnetic moments and reach such a high transition temperature (higher than room temperature $3[17-19$. This is obviously in contrast to the conventional magnetic theory based on Heisenberg's exchange mode 10 . Induced not by interaction but by unpaired $p_{z}$-electrons, the nonbonding states themselves cannot strongly interact with each other. Regarding the long-range and antisymmetric natures of the nonbonding state, combined with the experimental and theoretical observations $14|18| 19|27| 30 \mid 34$, we propose that the nonbonding state plays an important role in the ferromagnetism observed in graphene with vacancies. Due to its unpaired nature, the wavefunction of the state extends long range and keeps the orbital components on the atoms of the minority sublattice as little as possible. If the vacancy concentration in the minority sublattice of graphene is sufficiently large that the distances between the vacancies are smaller than the decay-length of the nonbonding states, the nonbonding-state-involved regions can be overlap. The induced nonbonding states could then be coherent in order to keep their orbital components on the atoms of minority sublattice as little as possible. Nonbonding states coupled in this way would maintain antisymmetric spatial wavefunctions, leading to ferromagnetic ordering.

These states (labeled by $s p^{2 *}$ and $p_{z}^{*}$ in the present work) contributing to magentism were also reported in the previous investigations $22|23| 26|27| 30|32| 41]$. In these papers, however, the magnetism of these states was attributed to the localization (for the unsaturated dangling bond states due to the so-called Jahn-Teller distortion (JTD)) according to the Hund's rule and to the itinerant electron (for the zero-mode states due to unpairing pielectrons between two sublattices) according to the itinerant electron mode, see e.g. the review $\operatorname{article}^{2}$. In contrast, the present work, for the first time, traced the magnetism in nonmagnetic materials to the antisymmetric manner of the spatial wavefunctions of the involved electronic states according to the electron exchange antisymmetric principle of quantum mechanics: if a spatial wavefunction of an electronic state is antisymmetric, its spin wavefunction must be symmetric. We believe this undebatable principle can end a long history of dispute about the origin of magnetism in nonmagnetic materials.

\section{CALCULATION METHODS}

The analyses were performed using our first principles calculations concerning the frame work of the spin density functional theory as implemented in the Vienna Ab initio Simulation Package (VASP) ${ }^{35}$. Electronion interactions were described using the projector augmented plane wave method 36 . It is well established that the generalized gradient approximation (GGA) of exchange-correlation functional is favorable for treating systems with non-uniform charge densities such as graphene. Therefore, the Perdew-Burke-Ernzerhof form of $\mathrm{GGA}^{37}$ was adopted in our calculations. In addition, we tested several cases using the local spin density functional (LSDA) for exchange-correlation effects 38 , which did not change the conclusions obtained with GGA. The wave functions were expanded in a plane wave basis with an energy cutoff of $500 \mathrm{eV}$ throughout the calculations. Two-dimensional Brillouin-zone (BZ) integrals were sampled on k-meshes corresponding to $48 \times 48$ and $96 \times 96$ in a $1 \times 1$-sized graphene cell when calculating the total energy and the density of states (DOS), respectively. The equilibrium lattice constant obtained via total energy minimization was $2.468 \AA$ for graphene, in good agreement with the experimental value, $2.46 \AA^{39}$. The systems were modeled as supercells with a vacuum of approximately $20 \AA$. All atoms in the slab were allowed to relax until the Hellmann-Feynman forces on the atoms were smaller than $0.01 \mathrm{eV} / \AA$. This calculation setup was found to be sufficiently accurate for our study. The maximally local- 
ized Wannier function process implemented in Wannier90 package ${ }^{40}$ was performed to obtain the orbital information for the relevant electronic structures.

\section{RESULTS AND DISCUSSION}

\section{A. A short summary and statements for the present study}

The main results (the MM and the long and short distance between three atoms surrounding the vacancy in the ground state) of graphene with a single vacancy in different vacancy concentration (for $2 \times 2 \sim 8 \times 8$-sized unit cells) are summarized in Table I. The MM for the cases vary over a range of 1.0 and $1.6 \mathrm{Bohr}$ magneton $\left(\mu_{\mathrm{B}}\right)$ and reach a stable value in the $7 \times 7$-sized cell, in good agreement with the corresponding results of previous calculations $22[27 / 30$.

TABLE I: Magnetic moments (MM) and structural parameters SD (short distance) and LD (long distance) describing the Jahn-Teller distortion for graphene with a vacancy in $2 \times 2$ $\sim 8 \times 8$-sized cell.

\begin{tabular}{cccccccc}
\hline \hline Size cell & $2 \times 2$ & $3 \times 3$ & $4 \times 4$ & $5 \times 5$ & $6 \times 6$ & $7 \times 7$ & $8 \times 8$ \\
\hline $\mathrm{MM}\left(\mu_{B}\right)$ & 1.0 & 1.1 & 1.6 & 1.6 & 1.6 & 1.3 & 1.3 \\
$\mathrm{SD}(\AA)$ & 2.59 & 2.56 & 2.18 & 2.11 & 2.05 & 1.99 & 1.97 \\
$\mathrm{LD}(\AA)$ & 2.59 & 2.56 & 2.58 & 2.58 & 2.58 & 2.57 & 2.57 \\
\hline \hline
\end{tabular}

In Table I, the JTD can be identified by the long and short sides of the isosceles triangle formed by the three atoms surrounding the vacancy. If the lengths of the long and short sides are equal, there is no JTD. Cases with cell sizes of $2 \times 2$ and $3 \times 3$ do not have JTD. To check whether JTD occurs in these cases, the symmetry of $C_{3 \mathrm{~V}}$ was destroyed by an artificial distortion (the length of the short and long sides being $1.40 \AA$ and $2.25 \AA$, respectively) as the initial atomic structure in the calculations. However, the optimized structures for the cases of $2 \times 2$ and $3 \times 3$ show that the differences between the short and long side disappear within our calculation accuracy, indicating that these two cases energetically favor keeping the $C_{3 \mathrm{~V}}$ symmetry without JTD. It can be understood because within a small sized cell, the obtained energy due to JTD cannot compensate for the energy lost due to strain. The magnetic moments exist for all the investigated cases, varying between $1.0 \mu_{\mathrm{B}}$ and $1.6 \mu_{\mathrm{B}}$, indicating that the magnetism in graphene with vacancies does not depend on the existence of JTD.

Even though the vacancy concentrations in which magnetism in graphene can be experimentally observed ${ }^{13 / 29}$ were much smaller than our investigated $2 \times 2$ and $3 \times 3$ sized cells, we would still take them into account to reveal the origin of the magnetism, because there is a structural turning point (with or without JTD). The JTD essentially does not affect the $p_{z}$ states but does significantly affect the $s p^{2}$ states relative to interactions between dangling bonds. The JTD-induced $s p^{2}$ dangling bond state has been understood in previous studies $\frac{1623 / 27 / 41}{\text { as an }}$ origin of magnetism in graphene with vacancies, that is, the $s p^{2}$ dangling bond state, which is induced by JTD in graphene with vacancies, contributed $1 \mu_{\mathrm{B}}$ according to Hund's rule because the dangling bond state looks like the state of an isolated atom. However, we will show later that, even if no JTD exists for the $2 \times 2$ and $3 \times 3$ sized cases, the $s p^{2}$ states of the three atoms surrounding the vacancy also contribute to the magnetism with quite different mechanism from that in $d f$-electron magnets. From the evolution of the MM relative to the electronic states near the turning point, the origin of the magnetism in the materials with only $s p$-electrons can be unambiguously understood, as discussed in more detail later. Therefore, the cases of the $2 \times 2$ and $3 \times 3$ sized cells are also considered in this study.

Note that all the atomic configurations listed in Table I are planar. Even though it has also been reported from first principles calculations ${ }^{42}$ that a nonplanar metastable state exists, we will not treat this state because we are focusing only on the origin of magnetism of $s p$-electrons, the planar configurations are in the ground state and can already be used to reveal the origin of the magnetism in nonmagnetic materials.

We will not perform calculations for the exchange energy based on any conventional magnetic models. This is because the vacancy concentration in proton-irradiated graphene, in which ferromagnetism has been observed, corresponds to a large distance between vacancies. A suitable simulation for this system requires a large supercell and is computationally intensive. In addition, such a long-range coupling is beyond any current magnetic models. The observed magnetization in the protonirradiated graphene is three or four orders of magnitude smaller than that of conventional magnets but with a high transition temperature (higher than room temperature). This is very difficult to be understood using the conventional magnetic theory. In other words, this type of ferromagnetism must have a distinctly different mechanism than that of conventional ferromagnetism. Significant conclusions could not be obtained from calculations based on the conventional magnetic theory; therefore, according to the nature of the nonbonding states, we did not perform calculations but rather postulated a possible ferromagnetic mechanism that is distinctly different from conventional mechanisms.

\section{B. Graphene with a vacancy in a $2 \times 2$-sized cell}

It is instructive to start the analysis of graphene in $2 \times 2$-sized cell with a single-atom vacancy, because its magnetism is almost entirely derived from a $p_{z}$ state near the Fermi level, i.e. $0.9 \mu_{\mathrm{B}}$ of the total $1.0 \mu_{\mathrm{B}}$ per vacancy, which is favorable to revealing the origin of the magnetism within $s p$-electron materials. The main magnetic 


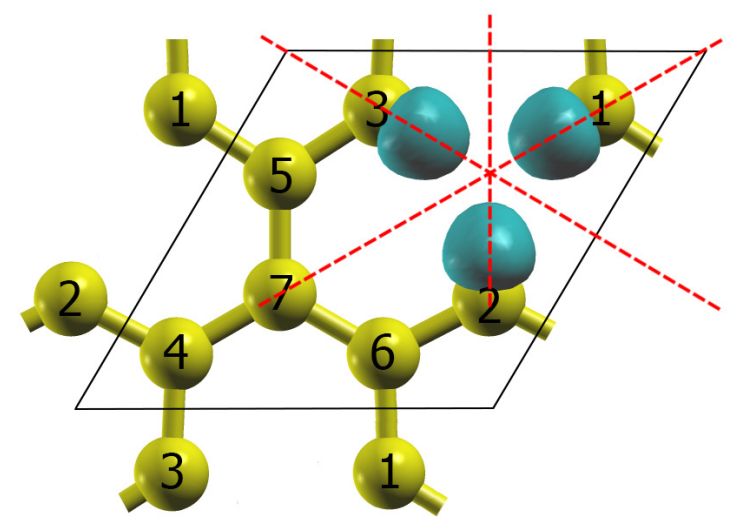

FIG. 1: (Color online) Ball and bond model for graphene with a vacancy in a $2 \times 2$-sized unit cell whose boundary is indicated by the solid lines. The three dashed lines represent the three mirror planes for the unit cell without John-Teller distortion. The gray region schematically indicates charge distribution of the $s p^{2}$-type dangling bonds on atoms 1,2 and 3 .

contribution for the other cases $(3 \times 3 \sim 8 \times 8)$ switches from the $p_{z}$ states to an $s p^{2}$ state of the dangling bonds. However, for the $2 \times 2$ case, the $s p^{2}$ state contributes little to the magnetism, $0.1 \mu_{\mathrm{B}}$ of $1.0 \mu_{\mathrm{B}}$.

The atomic structure of the $2 \times 2$-sized cell of seven carbon atoms, $g-\mathrm{C}_{7}$, is shown in Fig. 11. Atoms surrounding the vacancy for the other cases $(3 \times 3 \sim 8 \times 8)$ are similar but with a Jahn-Teller distortion for the $4 \times 4$-sized cell and larger. The atomic arrangement away from the vacancy can be regarded as a structural extension of perfect graphene.

The DOS and band structures for $g$ - $\mathrm{C}_{7}$ are shown in Figs. 2 (a) and 2(b), respectively. Due to the planar atomic structure, the $p_{z}$ states and the $s p^{2}$ hybridized states are orthogonal without interaction so that the $p_{z}$ and $s p^{2}$ orbital bands can be identified in Fig. 2 (b) by the colors red and blue, respectively; those contributing to the magnetism are labeled $p_{z}^{*}$ and $s p^{2 *}$, respectively, in Fig. 2 (a). The orbital types of these states were obtained via our orbital analysis based on the wavefunction projection method ${ }^{35}$, as well as on the maximally localized Wannier functions 40 . In Fig. 2 (b), the majority and minority bands are shown by the solid and dashed curves, respectively. For comparison, the DOS for graphene without a vacancy in the same sized cell as $g-\mathrm{C}_{7}$ is given by the red dashed curves in Fig. 2 (a).

Comparing the two DOSs (the black solids and red dashed lines) in Fig. 2 (a), the electronic structures of graphene near the Fermi level are shown to be largely distorted by the vacancy, leading to the magnetism in $g$ $\mathrm{C}_{7}$. The typical features (in the energy regions $-4 \mathrm{eV}$ and $+4 \mathrm{eV}$ ) of the electronic structures of graphene, which are characterized by the so-called Dirac point formed by the contact of the $\pi$ - and $\pi^{*}$-bands near the Fermi leve ${ }^{39}$, disappear due to the removal of one atom (the vacancy). Instead, three red and three blue bands as defect states

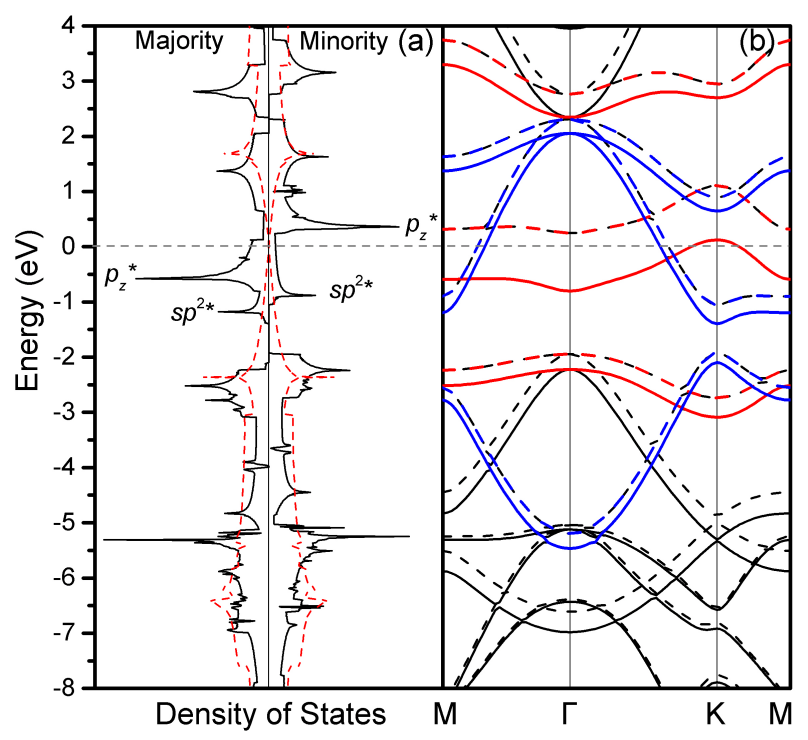

FIG. 2: (Color online) (a) DOS and (b) band structures of $g$ $\mathrm{C}_{7}$. Majority and minority bands in panel (b) are represented by solid and dashed lines, respectively. The MM involved in the blue and red bands in panel (b) consist of $s p^{2}$ and $p_{z}$ orbitals, respectively, labeled by $s p^{2 *}$ and $p_{z}^{*}$ in panel (a), respectively. The Fermi level is set to zero. For comparison, the DOS for perfect graphene in the same sized cell are shown by dashed curves in panel (a).

appear in this energy region. The magnetism of $g-\mathrm{C}_{7}$ can be traced to these defect states near the Fermi level. The $s p^{2 *}$ peaks for the majority and minority electrons with small spin splitting $(0.3 \mathrm{eV})$ lie below the Fermi level, and only the tails of the majority and minority near the Fermi level show a small difference, contributing only $0.1 \mu_{\mathrm{B}}$ of $1.0 \mu_{\mathrm{B}}$ per vacancy. Conversely, the spin splitting of the red state bands near the Fermi level is approximately $1 \mathrm{eV}$, and its majority part is nearly fully below the Fermi level, only a small party near the $\mathrm{K}$ point is above the Fermi level, while its minority part is above the Fermi level and unoccupied. Consequently, they contribute the majority of the MM for $g-\mathrm{C}_{7}, 0.9 \mu_{\mathrm{B}}$ of $1.0 \mu_{\mathrm{B}}$.

Our orbital analysis indicates that the red band near the Fermi level primarily consists of the $p_{z}$ orbitals of atoms 1, 2, 3 and 7, which belong to the same sublattice of graphene (referred to as sublattice A or the majority sublattice), while the removed atom (vacancy) belongs to the other sublattice (referred to as sublattice B or the minority sublattice), as do atoms 4,5 and 6 , which contribute little to the red states near the Fermi level. According to the symmetry of the atoms surrounding the vacancy, as shown in Fig. 1, the atoms contributing to the red band can be divided into two atom groups: atom 7 (A7) and atoms 1, 2 and 3 (A123). The orbital analysis also indicates that the red band consists of the two atom groups (A7 and A123) in an antisymmetric manner with 

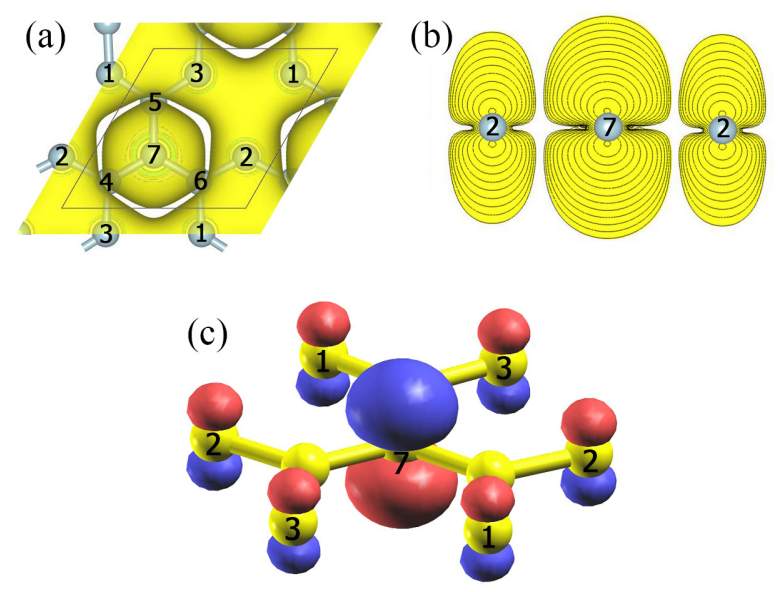

FIG. 3: (Color online) Charge distribution for the red band near the Fermi level of Fig. 2 (b) at $\Gamma$. (a) an isosurface as a topview; (b) a contour plot of the sideview for the vertical plane cutting along the line linking atoms 2-7-2 in (a)and (c) a schematic description for the antisymmetric orbital combination of the state on atoms 1, 2, 3 and 7 (lobe colors up-red and down-blue, as well as up-blue and down-red, indicate the opposite phases of the $p_{z}$ orbital). Yellow balls and stick indicate atoms and $s p^{2} \sigma$ bonds, respectively.

opposite phases as shown by the colors red and blue in Fig. 3 (c). Clearly, a node plane exists thus between the two atom groups, as shown by the contour plots in Figs. 3 (a) and 3(b). In the literature this state (the red band near the Fermi level) is referred to as the zero mode, implying a zero binding energy 22130 , while in molecular orbital theory (MOT), it is referred to as the nonbonding state $^{43}$, because its eigenvalue equals to the energy of the isolated $p_{z}$ orbital, denoted $p_{z}^{*}$ in the following. The $p_{z}^{*}$ state consisting of the $p_{z}$-orbitals of the two atom groups unexpectedly has no binding energy.

Due to its importance, let us take a closer look at the unexpected nonbonding state to illustrate its properties. For simplicity, a tight-binding method (TB) is applied to $g-\mathrm{C}_{7}$. In the TB model, each atom has only one $p_{z}$ orbital and only the first nearest neighbor (1NN) interaction is taken into account. Its Hamiltonian at $\Gamma$ is therefore

$$
\left(\begin{array}{ccccccc}
E_{0} & 0 & 0 & 0 & -t & -t & 0 \\
0 & E_{0} & 0 & -t & 0 & -t & 0 \\
0 & 0 & E_{0} & -t & -t & 0 & 0 \\
0 & -t & -t & E_{0} & 0 & 0 & -t \\
-t & 0 & -t & 0 & E_{0} & 0 & -t \\
-t & -t & 0 & 0 & 0 & E_{0} & -t \\
0 & 0 & 0 & -t & -t & -t & E_{0}
\end{array}\right) .
$$

Here $E_{0}$ and $t$ are an on-site energy of the $p_{z}$ orbital and a hopping energy between the nearest neighbors, respectively. The eigenvalue of the nonbonding state is $E_{\text {nonbonding }}=E_{0}$, and the eigenfunction is $(0.5,0.5,0.5$, $0.0,0.0,0.0,-1.0)$ ignoring the normalization coefficients.
That is, it is a state with zero binding energy, consisting only of the orbitals of the atom-groups A123 and A7 in an antisymmetric manner, without any contribution from atom-group B456, indicating that the nonbonding state is created by unpaired $p_{z}$ orbitals of atom-groups A123 and A7, not by a direct interaction between them because only the 1NN interaction was considered. Even though the state has a zero binding energy, occupying the nonbonding state still tends to stabilize the system as a way for electrons to synchronously appear in the two atom groups.

Most importantly, the nonbonding state consists of orbitals from A123 and A7 in an antisymmetric manner. This antisymmetric combination can be understood in the following way: if the interaction exists only between the nearest neighbors, no direct hopping exists between A123 and A7. Because there is direct hopping between A123 and B456, as well as between A7 and B456, the interaction for the nonbonding state could be thought of a hopping bridged via B456. Therefore, the hopping between A123 and A7 with a zero binding energy can be realized via the B456 bridge. We call this bridge hopping. However, because there are no real electrons on B456 for the nonbonding state, the wavefunction of the bridge hopping state should consist of orbitals from two involved atom-groups in an antisymmetric manner. This is the physics behind a state without any direct interaction but with an antisymmetric constraint for its wavefunction, as shown in Figs. 3 (b) and 3(c). Note that the nonbonding state here should be distinguished from a lone pair state of a dangling bond. A lone pair state of a dangling bond does not involve with any atoms except for itself. However, the nonbonding states involve at least atom-groups A123 and A7, leading therefore to an antisymmetric combination of the orbitals of the involved atoms.

We have seen that the MM in $g$ - $\mathrm{C}_{7}$ should primarily be traced to the red band (the $p_{z}^{*}$ nonbonding state) with a small component traced to the blue band $\left(s p^{2 *}\right)$ near the Fermi level. The $s p^{2 *}$ state will be discussed in more detail in the next subsection; here we list its main features. The orbital analysis indicates that the $s p^{2 *}$ state arises from the interaction between the $s p^{2}$ dangling bonds of the three atoms surrounding the vacancy. The three bands with the features of the $s p^{2}$ dangling bonds are identified by the color blue in Fig. 2(b). Only one of the three is a bonding state band, whose majority DOS peak lies at approximately $-2.8 \mathrm{eV}$, and the other two are antibonding states whose majority DOS peaks are at approximately $-1.2 \mathrm{eV}$ and $+1.4 \mathrm{eV}$. The two antibonding state bands degenerate at $\Gamma$. The three blue bands have dispersions of approximately $3.4 \mathrm{eV}, 3.5 \mathrm{eV}$ and $1.4 \mathrm{eV}$. Therefore, their DOS peaks are not very sharp.

It is well known that the nonbonding state and the antibonding state consist of antisymmetric orbitals. Thus, the MM of the $s p$ electrons in $g-\mathrm{C}_{7}$ can be understood. According to the electron exchange antisymmetric principle of quantum mechanics, if a spatial wavefunction of 


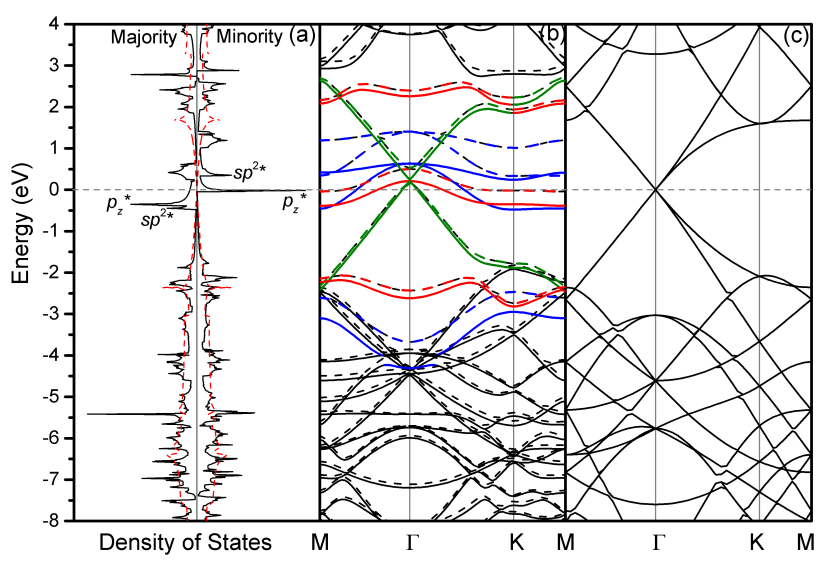

FIG. 4: (a) and (b) Same as that of Fig. 2 but for $g$-C $\mathrm{C}_{17}$. (c) the band structure of the graphene in the same sized cell for comparison. The features with the green bands in panel (b) appear in all $3 n \times 3 n$-sized cells of graphene with a vacancy, because the bands are nearly unaffected by the removed atom, see text for details.

a state is antisymmetric, its spin component should be symmetric 44 . It has been debated for a long time if $s p$ electrons could lead to MM in materials. The results of $g-\mathrm{C}_{7}$ show that the $\mathrm{MM}$ in $g-\mathrm{C}_{7}$, created by $s p$-electrons, can be traced to fundamental principle of quantum mechanics. This is the physics behind the magnetism of $s p$-electrons: it is not $s p$-orbitals themselves but the antisymmetric behavior of their spatial wavefunctions that leads to electrons in spin polarization. This mechanism is different from the magnetic origin of $d f$-electrons as proposed by Heisenberg ${ }^{[0}$, we will discuss in more detail in Subsection IIIF.

\section{Graphene with a vacancy in a $3 \times 3$-sized cell}

The calculated DOS and band structures for graphene with a vacancy in a $3 \times 3$-sized cell, $g-\mathrm{C}_{17}$, are shown in Figs. 4 (a) and 4(b), respectively. Again the red and blue bands indicate the states composed of the $p_{z}$ and $s p^{2}$ orbitals, respectively. For comparison, the band structures of perfect graphene in the same sized cell, $g$-3×3, are also shown in Fig. 4 (c); the structures have no spin polarization as expected.

At first glance, two green bands near the Fermi level in Fig. 4 (b) appear to be copied from the corresponding bands of $g-3 \times 3$ in Fig. 4 (c) and one Dirac point remains. That is, these two bands ( $\pi$ and $\pi^{*}$ ) of perfect graphene are nearly unperturbed by the removal of an atom (the vacancy) and contact each other to form a Dirac point. This is entirely different from the case of $g-\mathrm{C}_{7}$. In $g$ $\mathrm{C}_{7}$, the vacancy removes the two Dirac points (at $\mathrm{K}$ and $\mathrm{K}^{\prime}$ ), leading to a formal gap near the Fermi level; as we discussed, all states of $g-\mathrm{C}_{7}$ in the formal gap region can

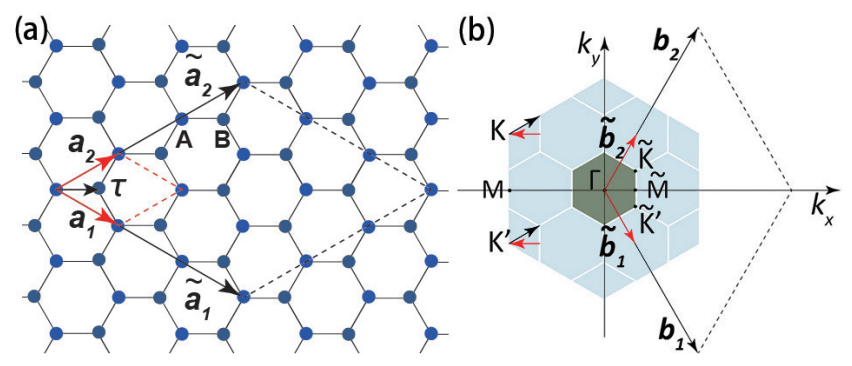

FIG. 5: The relationship of (a) the unit cells and (b) the Brillouin zones for the $1 \times 1$ - and $3 \times 3$-sized cells. Vectors a and $\mathbf{b}$ are basis vectors for the lattice and the reciprocal lattice, respectively. The tilde on the symbols indicate values for the $3 \times 3$-sized cell.

be traced to the vacancy-induced defect states. Clearly, the one Dirac point (formed by the two green bands) in Fig. 4 (b) is not affected by the removal of an atom.

We know that the Dirac points ${ }^{39}$ at $\mathrm{K}$ and $\mathrm{K}^{\prime}$ for $g-1 \times 1$ disappear in $g-\mathrm{C}_{7}$ because the vacancy disturbs the network of the $p_{z}$-orbitals of graphene, therefore destroying the Dirac points at $K$ and $K$ '. It is natural to expect this feature (the disappearance of the Dirac points) to remain when such cells are expanded. However, it is not true for the cases of $3 n \times 3 n$-sized cells (where $n$ is an integer). The natural question is, why not?

We then take $g-\mathrm{C}_{17}$ as an example to answer this question. In $g-3 \times 3$, the Dirac point (the two bands contact each other) appears not at $\mathrm{K}$ and $\mathrm{K}$ ', but degenerated at the $\Gamma$ point of the $3 \times 3 \mathrm{BZ}$. This is expected because of the so-called BZ folding. The relationship of the unit cells and BZs of graphene between the $1 \times 1$ - and $3 \times 3$ sized cells is shown in Figs. 5 (a) and 5(b), respectively. Clearly, the $\mathrm{K}$ and $\mathrm{K}^{\prime}$ points of the $1 \times 1 \mathrm{BZ}$ are at the $\Gamma$ point of the $3 \times 3 \mathrm{BZ}$.

The folding relationship can be also obtained by considering the relationship of the primitive vectors between the $3 \times 3$ - and $1 \times 1$-sized cells. The corresponding primitive vectors of the $3 \times 3$-sized cell, $\widetilde{\mathbf{a}}_{1}$ and $\widetilde{\mathbf{a}}_{2}$, are three times those of the $1 \times 1$-sized cell, $\mathbf{a}_{1}$ and $\mathbf{a}_{2}$, i.e., $\widetilde{\mathbf{a}}_{1}=$ $3 \mathbf{a}_{1}$, and $\widetilde{\mathbf{a}}_{2}=3 \mathbf{a}_{2}$. Therefore, the relationship of the primitive vectors of the reciprocal lattice between the two lattices should be $\widetilde{\mathbf{b}}_{1}=1 / 3 \mathbf{b}_{1}$ and $\widetilde{\mathbf{b}}_{2}=1 / 3 \mathbf{b}_{2}$. The $\mathrm{K}$ and $\mathrm{K}^{\prime}$ points for the $1 \times 1$-sized cell can be represented by $\mathbf{b}_{1}$ and $\mathbf{b}_{2}$ as $\mathbf{K}=2 / 3 \mathbf{b}_{1}+1 / 3 \mathbf{b}_{2}$ and $\mathbf{K}^{\prime}=1 / 3 \mathbf{b}_{1}+2 / 3 \mathbf{b}_{2}$. Therefore, by means of the primitive vectors of the reciprocal lattice for the $3 \times 3$-sized cell, $\widetilde{\mathbf{b}}_{1}$ and $\widetilde{\mathbf{b}}_{2}$, they should be written as $\mathbf{K}=2 / 3 \mathbf{b}_{1}+1 / 3 \mathbf{b}_{2}=2 \widetilde{\mathbf{b}}_{1}+\widetilde{\mathbf{b}}_{2}$ and $\mathbf{K}^{\prime}=1 / 3 \mathbf{b}_{1}+2 / 3 \mathbf{b}_{2}=\widetilde{\mathbf{b}}_{1}+2 \widetilde{\mathbf{b}}_{2} . \mathbf{K}$ and $\mathbf{K}^{\prime}$ are the two reciprocal lattice vectors for the $3 \times 3$-size cell. One end-point of a reciprocal lattice vector is the center of its Brillouin zone, the $\Gamma$ point. That is, both $\mathrm{K}$ and $\mathrm{K}$ ' of the $1 \times 1$-sized cell are folded to and degenerated at one point, the $\Gamma$ point of the $3 \times 3$-sized cell. This is also valid for all cells with sizes of $3 n \times 3 n$.

The Dirac point for $g-\mathrm{C}_{17}$ is fourfold degenerated be- 
cause both the $\mathrm{K}$ and $\mathrm{K}^{\prime}$ point of the $1 \times 1 \mathrm{BZ}$ are folded to the $\Gamma$ point of the $3 \times 3 \mathrm{BZ}$. Furthermore, it can be seen from Fig. 4 (c) that, along the $\mathrm{M}-\Gamma$ axis, the bands of $g-3 \times 3$ just below and above the Fermi level are degenerated while along the $\Gamma-K$ axis, the degeneration of the bands is lifted because the two axes pointed by two red arrows (from $\widetilde{\mathrm{M}}$ to $\widetilde{\Gamma}$ of the $3 \times 3 \mathrm{BZ}$ ) are equivalent in the $1 \times 1 \mathrm{BZ}$, while that of the two black arrows (from $\widetilde{\Gamma}$ to $\widetilde{\mathrm{K}}$ of the $3 \times 3 \mathrm{BZ}$ ) are not, as shown in Fig. 5 (b).

Keeping this relationship in mind, we can now understand why the one Dirac point does not disappear even if one atom is removed from $g-3 \times 3$ and two green bands in Fig. 5 (b) resemble the corresponding bands in $g-3 \times 3$. For this discussion, we again use the TB model. In the
$1 \times 1$-sized cell of graphene, the Bloch sums at sites $\mathrm{A}$ and B (labeled in Fig. 5 (a)) are

$$
\psi_{\mathbf{k}}^{A}=\sum_{\mathbf{R}} \phi^{A}(\mathbf{r}-\mathbf{R}) e^{i \mathbf{k} \cdot \mathbf{R}}
$$

and

$$
\psi_{\mathbf{k}}^{B}=\sum_{\mathbf{R}} \phi^{B}(\mathbf{r}-\mathbf{R}-\boldsymbol{\tau}) e^{i \mathbf{k} \cdot(\mathbf{R}+\boldsymbol{\tau})}
$$

respectively. They form the so-called $\pi$ and $\pi^{*}$ states in the symmetric and antisymmetric types, respectively, (ignoring the normalization coefficients) as

$$
\psi_{\mathbf{k}}^{\pi}=\sum_{\mathbf{R}} \phi^{A}(\mathbf{r}-\mathbf{R}) e^{i \mathbf{k} \cdot \mathbf{R}}+C_{\mathbf{k}} \sum_{\mathbf{R}} \phi^{B}(\mathbf{r}-\mathbf{R}-\boldsymbol{\tau}) e^{i \mathbf{k} \cdot(\mathbf{R}+\boldsymbol{\tau})}
$$

and

$$
\psi_{\mathbf{k}}^{\pi^{*}}=\sum_{\mathbf{R}} \phi^{A}(\mathbf{r}-\mathbf{R}) e^{i \mathbf{k} \cdot \mathbf{R}}-C_{\mathbf{k}} \sum_{\mathbf{R}} \phi^{B}(\mathbf{r}-\mathbf{R}-\boldsymbol{\tau}) e^{i \mathbf{k} \cdot(\mathbf{R}+\boldsymbol{\tau})} .
$$

Because the $3 \times 3$-sized cell is only artificially extended from $1 \times 1$-sized cell, the bands' properties (bonding and antibonding) should be maintained, the $\pi$ and $\pi^{*}$ bands along the M- $\Gamma$ axis for $g-3 \times 3$ should still be composed of the $\pi$ and $\pi^{*}$ in $g-1 \times 1$. For the BZ relationship between $g$ - $1 \times 1$ and $g-3 \times 3$, please refer to Fig. 5 . $\tau$ has no $y$ component, and because $\mathbf{k}$ and $\mathbf{k}^{\prime}$ in $g-1 \times 1$ are symmetric in the $x$-direction, as shown in Fig. 5(b); therefore, along the $\mathrm{M}-\Gamma$ of $g-3 \times 3$, we obtain

$$
\begin{aligned}
\Psi_{A}^{\pi} & =\psi_{\mathbf{K}+\Delta \mathbf{k}}^{\pi}-\psi_{\mathbf{K}^{\prime}+\Delta \mathbf{k}}^{\pi} \\
& =\sum_{\mathbf{R}} \phi^{A}(\mathbf{r}-\mathbf{R}) e^{i \Delta \mathbf{k} \cdot \mathbf{R}}\left(e^{i \mathbf{K} \cdot \mathbf{R}}-e^{i \mathbf{K}^{\prime} \cdot \mathbf{R}}\right) \\
& +\sum_{\mathbf{R}} e^{i\left(\frac{4 \pi}{3}+\Delta k_{x} a\right)} \phi^{B}(\mathbf{r}-\mathbf{R}-\boldsymbol{\tau}) e^{i \Delta \mathbf{k} \cdot \mathbf{R}}\left[C_{\mathbf{K}+\Delta \mathbf{k}} e^{i \mathbf{K} \cdot \mathbf{R}}-C_{\mathbf{K}^{\prime}+\Delta \mathbf{k}} e^{i \mathbf{K}^{\prime} \cdot \mathbf{R}}\right] \\
& =\sum_{\mathbf{R}\left(\neq \mathbf{R}^{\prime}\right)}\left[\phi^{A}(\mathbf{r}-\mathbf{R})+C_{\mathbf{K}+\boldsymbol{\Delta} \mathbf{k}} e^{i\left(\frac{4 \pi}{3}+\Delta k_{x} a\right)} \phi^{B}(\mathbf{r}-\mathbf{R}-\boldsymbol{\tau})\right] e^{i \Delta \mathbf{k} \cdot \mathbf{R}}\left(e^{i \mathbf{K} \cdot \mathbf{R}}-e^{i \mathbf{K}^{\prime} \cdot \mathbf{R}}\right)
\end{aligned}
$$

and

$$
\begin{aligned}
\Psi_{A}^{\pi^{*}} & =\psi_{\mathbf{K}+\boldsymbol{\Delta} \mathbf{k}}^{\pi^{*}}-\psi_{\mathbf{K}^{\prime}+\boldsymbol{\Delta} \mathbf{k}}^{\pi^{*}} \\
& =\sum_{\mathbf{R}\left(\neq \mathbf{R}^{\prime}\right)}\left[\phi^{A}(\mathbf{r}-\mathbf{R})-C_{\mathbf{K}+\boldsymbol{\Delta} \mathbf{k}} e^{i\left(\frac{4 \pi}{3}+\Delta k_{x} a\right)} \phi^{B}(\mathbf{r}-\mathbf{R}-\boldsymbol{\tau})\right] e^{i \Delta \mathbf{k} \cdot \mathbf{R}}\left(e^{i \mathbf{K} \cdot \mathbf{R}}-e^{i \mathbf{K} \cdot \mathbf{R}}\right)
\end{aligned}
$$


with $C_{\mathbf{K}+\Delta \mathbf{k}}=C_{\mathbf{K}^{\prime}+\Delta \mathbf{k}}$. The above sum of $\mathbf{R}$ excludes $\mathbf{R}^{\prime}$ because $\mathbf{K} \cdot \mathbf{R}=\mathbf{K}^{\prime} \cdot \mathbf{R}+2 \pi\left(n_{1}-n_{2}\right) / 3$ for all $\mathbf{R}^{\prime}$ lattice vectors satisfying $n_{1}-n_{2}=3 n$ with $n$ as an integer and $e^{i \mathbf{K} \cdot \mathbf{R}}-e^{i \mathbf{K}^{\prime} \cdot \mathbf{R}}=0$. Therefore, the two-fold degenerate bands along the $\mathrm{M}-\Gamma$ axis of $g-3 \times 3$ are independent of all lattice vectors $\mathbf{R}^{\prime}$. Therefore, removing one atom from graphene (creating a vacancy) in a $3 n \times 3 n$ sized cell will not significantly affect the one Dirac point and the involved bands (green) if it is modeled using the TB model.

Note that the green bands have nearly no spin polarization, as shown in Fig. 4 (b). Like the red bands near the Fermi level, the green bands also consist of the $p_{z}$ orbitals and also appear in the same energy region. Therefore, the reason that the green bands do not contribute to magnetism but the red bands do is not related to the intrinsic nature of the $p_{z}$ orbitals but to the antisymmetric type of the wavefunctions, indicating that, unlike $d f$ electrons, $s p$ electrons have only small amounts of exchange energy. No defect states (antibonding states or nonbonding states here) mean no magnetism for $s p$ electrons in an $s p$-bonded crystal. This may be why $s p$ electron magnetism has been in doubt for such a long time 6 8]15.

The $\mathrm{MM}$ of $g-\mathrm{C}_{7}$ are primarily derived from the $p_{z}^{*}$ nonbonding states, while the main magnetic contributor in $g-\mathrm{C}_{17}$ is the $s p^{2 *}$ blue bands near the Fermi level. Now, we focus on the blue bands in $g-\mathrm{C}_{17}$. The orbital analysis indicates that the blue bands consist of $s p^{2}$-type orbitals of the three $\mathrm{C}$ atoms surrounding the vacancy, atoms 1,2, and 3. From our previous investigations of $g-\mathrm{C}_{14} \mathrm{~N}_{3}{ }^{45 / 46}$ and $g-\mathrm{C}_{4} \mathrm{~N}_{3} \frac{47}{4}$, it is known that the dangling bonds of the three $\mathrm{N}$ atoms interact each other to form one bonding state and two antibonding states. The difference between $g-\mathrm{C}_{14} \mathrm{~N}_{3}\left(g-\mathrm{C}_{4} \mathrm{~N}_{3}\right)$ and $g$ - $\mathrm{C}_{17}\left(g-\mathrm{C}_{7}\right)$ is that the three $\mathrm{N}$ atoms surrounding the vacancy in $g-\mathrm{C}_{14} \mathrm{~N}_{3}\left(g-\mathrm{C}_{4} \mathrm{~N}_{3}\right)$ are replaced by three $\mathrm{C}$ atoms. The two antibonding states, degenerated at the $\Gamma$-point, have a mirror-symmetric combination (MSC) and a mirrorantisymmetric combination (MAC) of the $s p^{2}$ orbitals with $C_{3 \mathrm{~V}}$ symmetry. Examining $g$ - $\mathrm{C}_{17}\left(g-\mathrm{C}_{7}\right)$, the situation is similar to $g-\mathrm{C}_{14} \mathrm{~N}_{3}\left(g-\mathrm{C}_{4} \mathrm{~N}_{3}\right)^{45 / 46}$ : the three blue bands are also induced by the interaction between the three dangling bonds on the three atoms surrounding the vacancy. When one $\mathrm{C}$ atom is removed from graphene, the three $s p^{2}$ dangling bonds on the three atoms surrounding the vacancy are left. The dangling bonds, which were originally connected to the removed atom, are not fully filled. Therefore, they interact with each other and form one bonding state and two antibonding states.

To illustrate the resulting three (one bonding and two antibonding) states, a TB model is again taken into account. For three atoms each with only a dangling bond orbital, the Hamiltonian is

$$
\left(\begin{array}{ccc}
E_{0} & -t & -t \\
-t & E_{0} & -t \\
-t & -t & E_{0}
\end{array}\right) .
$$

(a)
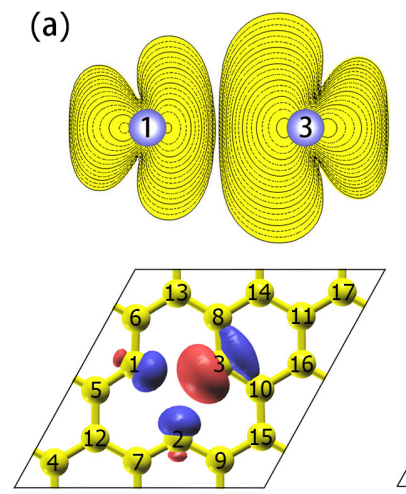

(b)
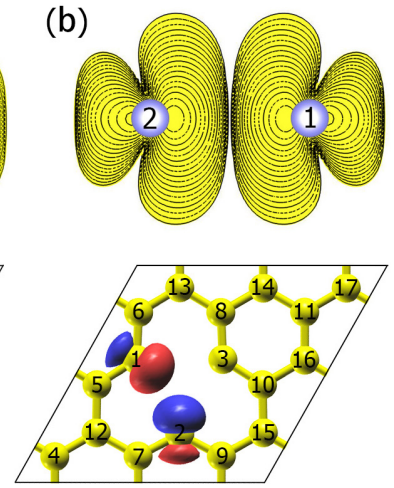

FIG. 6: Charge distribution for the red band in Fig. 4 (a) at $\Gamma$ for the (a) MSC and (b) for MAC states. Panels (a) and (b) are the up and down panels for the side and top views, respectively. In the side view, the vertical plane cuts along the line linking atoms 1-3 and atoms 1-2 for panels (a) and (b), respectively.

Here $E_{0}$ and $t$ are the energy of the dangling bond and the interaction between the dangling bonds, respectively. We can obtain the energy of the bonding state as $E_{1}=$ $E_{0}-2 t$ and those of the doublet degenerated antibonding states as $E_{2}=E_{3}=E_{0}+t$. The difference between $g-\mathrm{C}_{17}$ $\left(g-\mathrm{C}_{7}\right)$ and $g-\mathrm{C}_{14} \mathrm{~N}_{3}\left(g-\mathrm{C}_{4} \mathrm{~N}_{3}\right)$ is that the MSC and MAC bands are partially occupied in the cases of $g-\mathrm{C}_{17}$ and $g$ - $\mathrm{C}_{7}$, while in the cases of $g-\mathrm{C}_{4} \mathrm{~N}_{3}$ and $g-\mathrm{C}_{14} \mathrm{~N}_{3}$, both bands are nearly fully occupied. This is because $g-\mathrm{C}_{14} \mathrm{~N}_{3}$ $\left(g-\mathrm{C}_{4} \mathrm{~N}_{3}\right)$ has more three electrons than $g-\mathrm{C}_{17}\left(g-\mathrm{C}_{7}\right)$ and is a $+1 e$ hole system, while $g$ - $\mathrm{C}_{17}\left(g-\mathrm{C}_{7}\right)$ is a $+4 e$ hole system. Therefore, the Fermi level is lowered relative to the top of the MSC and MAC bands in $g-\mathrm{C}_{17}\left(g-\mathrm{C}_{7}\right)$.

The features of the MSC and MAC antibonding states in the charge distribution at $\Gamma$ are shown in Figs. 6 (a) and 6(b), respectively. The blue and red colors in Fig. 6 (b), as that in Fig. 3 (c), indicate the different phases, and nodes exists between atoms 1(2) and 3 for the MSC state, as well as between atoms 1 and 2 for the MAC state, as shown by the counter plots in Figs. 6 (a) and 6(b), indicating an antisymmetric combination of the involved orbitals on the three atoms.

Note that previous calculations attributed the magnetic contribution of the $s p^{2}$-type states in graphene with vacancies to $\mathrm{JTD} 16 / 2327141$. However, even without JTD in $g$ - $\mathrm{C}_{17}$ the $s p^{2 *}$ states contribute to the magnetism, indicating that the magnetism in graphene with vacancies is independent of JTD. The origin of the magnetism arising from the states can be also traced to the antisymmetric mode of their wavefunctions.

\section{Graphene with a vacancy in a $4 \times 4$-sized cell}

For cell sizes larger than $4 \times 4$, the optimized atomic structure for graphene with a vacancy has a JTD. That is, two of the three atoms surrounding the vacancy move 


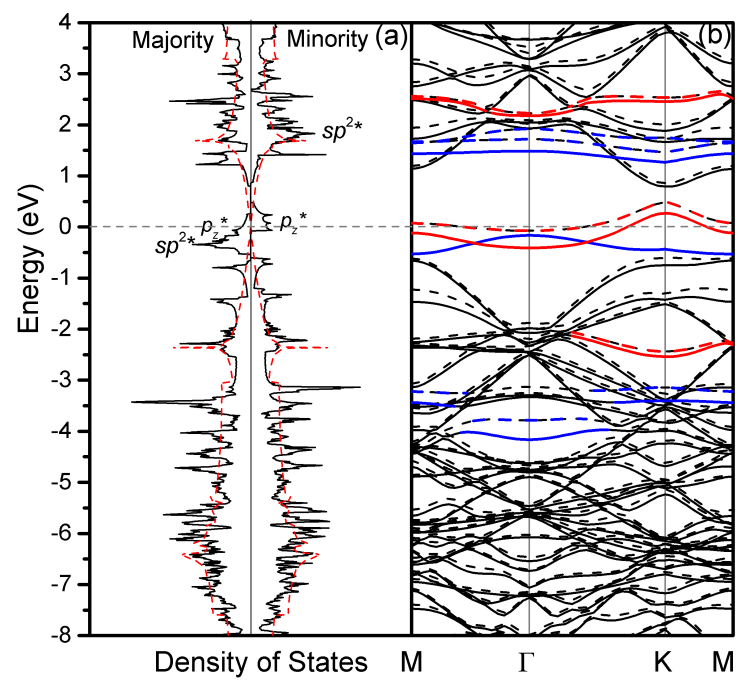

FIG. 7: The same as Fig. 2 and Fig. 4 but for $g$-C $\mathrm{C}_{31}$.

closer to each other, while the third one moves away, forming thus an isosceles triangle with one short and two long sides. The rest of the atoms in the unit cell experience only minor change due to JTD, whereas the relevant electronic structures are not significantly affected by the minor change.

As in $g$ - $\mathrm{C}_{7}$ and $g-\mathrm{C}_{17}$, the vacancy-induced $s p^{2}$ and $p_{z}$ bands are again shown in blue and red, respectively, in the band structure in Fig. 77(b). As shown in Fig. 7, the $p_{z}^{*}$-nonbonding state (red) bands, due to the unpaired $p_{z}$ orbitals of the atoms in the same sublattice, still appear near the Fermi level as expected. Because the $p_{z}$ and $s p^{2}$ orbitals are orthogonal, the nonbonding state bands are not significantly affected by JTD, contributing approximately $0.6 \mu_{\mathrm{B}}$, consistent with previous calculations (referred to as the zero mode states ${ }^{48 / 49}$. As discussed in $g-\mathrm{C}_{7}$ and $g-\mathrm{C}_{17}$, note that the important factor for the nonbonding states is not whether the binding energy is zero but its antisymmetric orbital combination, to which the magnetic contribution can be traced. The occupation of some minority nonbonding states can be traced to the competition between the $\mathbf{k}$-dependent kinetic energy and the exchange energy. The other two $p_{z}$ orbital bands, namely the $\pi$ - and $\pi^{*}$-bands (red), lie at approximately $-2.5 \mathrm{eV}$ and $2.5 \mathrm{eV}$ respectively, and do not contribute to the MM because their majority and minority bands are either fully occupied or completely empty.

The main contributor to the magnetism in $g$ - $\mathrm{C}_{31}$ is the blue bands near the Fermi level, as shown in Figs. 7 (a) and $7(\mathrm{~b})$; the majority blue band near the Fermi level is fully occupied, while the corresponding minority one $(\sim 1.8 \mathrm{eV})$ is completely empty, therefore contributing $1.0 \mu_{\mathrm{B}}$ to the magnetic moment of $g-\mathrm{C}_{31}$. The JTD parameters (long side length $2.58 \AA$ and short side $2.18 \AA$ ), the band dispersion and the contributing magnitude to the magnetic moment of the blue bands obtained by our calculations are in agreement with the corresponding calculations $23 \mid 27 / 48 / 49$. However, the explanation is different. It was suggested $\sqrt{23 / 27 / 42}$ that, due to JTD, two of the three atoms around the vacancy form a $s p^{2} \sigma$ bond, leaving the apical atom with a dangling bond contributing a magnetic moment of approximately $1.0 \mu_{\mathrm{B}}$ according to Hund's rule. However, because the short side is much larger than an $s p^{2} \sigma$ bond length in perfect graphene, $1.42 \AA^{39}$, it is impossible for the two atoms to form an $s p^{2} \sigma$ bond, leaving the apical atom alone to form a solitary dangling bond and to be filled by one electron with spin polarization. Even if a solitary dangling bond exists on one atom, the magnetism obtained by calculations cannot be traced to it because in the calculations based on the SEA, one electron can half fill on one spin channel and half on the other spin channel. A famous example contrary to the previous conclusion that the magnetism in graphene with vacancies is contributed to the dangling bond is the dangling bonds on the $\mathrm{Si}(111)$ surface. Dangling bond states exist on the $\mathrm{Si}(111)$ surface and their electron states satisfy the local condition. However, the $\mathrm{Si}(111)$ surface does not show any spin polarization ${ }^{50}$ because a half electron occupies the spin-up channel and the other half one can occupy the spin-down channel of the dangling bond state. Therefore, Hund's rule does not hold when interpreting results calculated based on SEA.

The vacancy-induced $s p^{2 *}$ states are formed by the interaction between all three atoms surrounding the vacancy, forming one bonding and two antibonding states and identified by the color blue in Fig. 7(b). Even though the $C_{3 \mathrm{~V}}$ symmetry in $g-\mathrm{C}_{31}$ is broken due to JTD, two of the three blue bands, which consist of the $s p^{2}$ orbitals of the three atoms around the vacancy and lie near the Fermi level and $\sim+1.5 \mathrm{eV}$, respectively, can still be characterized by MSC and MAC. This is a key to understanding the magnetism in graphene with vacancies. That is, all states that contribute to the magnetism consist of antisymmetric spatial wavefunctions. When the states appear near the Fermi level, they are partially occupied in spin polarization according to the electron exchange antisymmetric principle of quantum mechanics. This is a different mechanism than that suggested by Heisenberg for $d f$-electrons $\frac{10}{10}$, which we will discuss later.

To further illustrate the antisymmetric nature of MSC, we built a TB model of three $s p^{2}$ dangling bonds on three atoms with a JTD; only one $s p^{2}$ dangling bond for each atom of the three atoms was taken into account. The Hamiltonian of the TB model can be easily written as

$$
\left(\begin{array}{ccc}
E_{0} & -t_{1} & -t_{1} \\
-t_{1} & E_{0} & -t_{2} \\
-t_{1} & -t_{2} & E_{0}
\end{array}\right) .
$$

Here $t_{1}$ and $t_{2}$ are the interactions between two atoms on the long and short sides of an isosceles triangle, respectively, with $t_{2}>t_{1}$. One can obtain $E_{1}=E_{0}-$ $\frac{1}{2}\left(t_{2}+\sqrt{t_{2}^{2}+8 t_{1}^{2}}\right), E_{2}=E_{0}+\frac{1}{2}\left(\sqrt{t_{2}^{2}+8 t_{1}^{2}}-t_{2}\right)$ and 


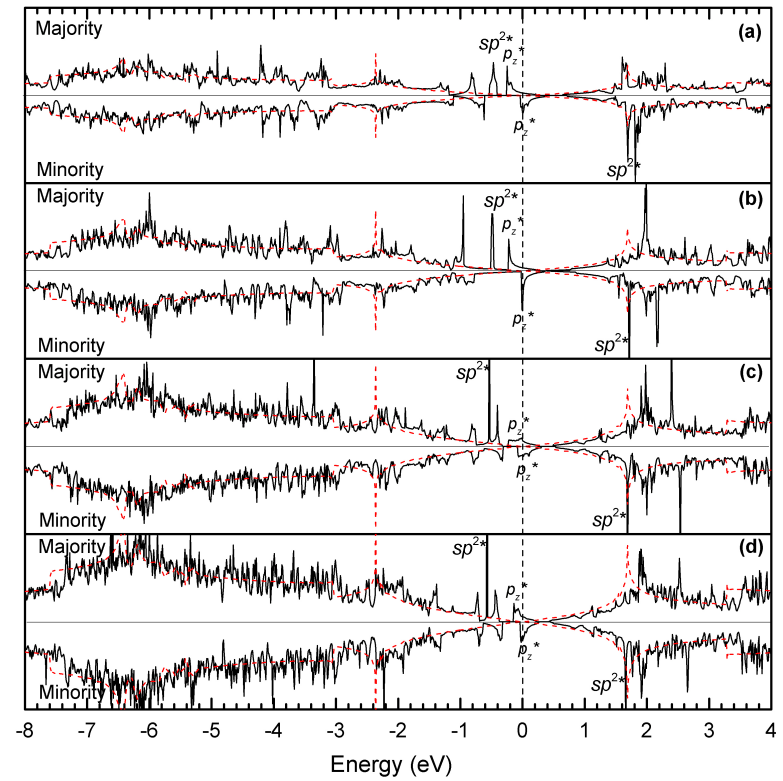

FIG. 8: Density of states for graphene with a vacancy in $5 \times 5$ (a), $6 \times 6$ (b), $7 \times 7$ (c) and $8 \times 8$ (d) size. The symbols of $s p^{2 *}$ and $p_{z}^{*}$ have the same orbital type as in Figs. 2, 4 and 7

$E_{3}=E_{0}+t_{2}$. The corresponding eigenfunctions for $E_{1}$, $E_{2}$ and $E_{3}$ are $\left(\frac{-t_{2}+\sqrt{8 t_{1}^{2}+t_{2}^{2}}}{2 t_{1}}, 1,1\right),\left(\frac{-t_{2}-\sqrt{8 t_{1}^{2}+t_{2}^{2}}}{2 t_{1}}, 1,1\right)$ and $(0,-1,1)$, respectively, clearly showing the antisymmetric combination for $E_{2}$ and $E_{3}$. This means that, if the three unsaturated dangling bonds interact with each other, they would form one bonding state with a symmetric orbital combination and two antibonding states each with an antisymmetric orbital combination. Clearly, the two antibonding states, $E_{2}$ and $E_{3}$, correspond to MSC and MAC respectively, while $E_{1}$ corresponds to the $s p^{2}$ bonding state.

Therefore, we can understand the magnetism contributed by the MSC state: JTD breaks the constrain of the $C_{3 \mathrm{~V}}$ symmetry, therefore lifting the degeneration of MSC and MAC at $\Gamma$. The MSC $s p^{2 *}$ band (blue) is therefore lower in energy than the $p_{z}^{*}$-nonbonding state due to $s$-orbital components in the $s p^{2 *}$ states; the majority of the MSC band is fully filled by one electron, leaving its minority band completely empty and contributing $1.0 \mu_{\mathrm{B}}$ according to the electron exchange antisymmetric principle of the quantum mechanics.

\section{E. Graphene with a vacancy in $5 \times 5 \sim 8 \times 8$-sized cells}

Electronic structures for $5 \times 5 \sim 8 \times 8$-sized cells of graphene with a vacancy are similar to the corresponding structures for the $2 \times 2 \sim 4 \times 4$-sized cells. Importantly, the MM in these cases are derived from the antibonding and nonbonding states. Here we briefly discuss the magnetism-concerned electronic structures in the $5 \times 5 \sim$ $8 \times 8$-sized cells by means of DOS, as shown in Figs. 8 (a) $\sim 8(\mathrm{~d})$.

Similar to the $4 \times 4$ case, the peaks of the majority antibonding states (labeled by $s p^{2 *}$ as shown in Fig. 8 lie at approximately $-0.6 \mathrm{eV}$ for the involved cases $(5 \times 5 \sim$ $8 \times 8)$ and are fully occupied, while their minority peaks lie at approximately $+1.6 \mathrm{eV}$ and are completely empty. Therefore, the $s p^{2 *}$-antibonding states contribute a magnetic moment of $1.0 \mu_{\mathrm{B}}$. These peaks become increasingly sharper with increasing cell sizes, implying that the dispersion of the corresponding bands decrease. It is expected that the dispersions of the bands depend on the interaction of the $s p^{2 *}$-antibonding states over the unit cell; this interaction is therefore increasingly weaker as the cell sizes increase.

Conversely, the peak width of the $p_{z}^{*}$-nonbonding states, which lie around -0.2 and $\sim 0.0 \mathrm{eV}$ for the majority and minority, respectively, and contribute to $\mathrm{MM}$ of $0.6 \sim 0.3 \mu_{\mathrm{B}}$, does not depend as much on the cell sizes because their wavefunctions are widely extended, decaying with the distance $r$ to the vacancy as $\sim 1 / r^{27}$. This feature can be understood because these states are induced by the unpaired $p_{z}$ orbitals, involving not only two atoms but also additional atoms in the majority sublattice of the unit cell. This is a very important feature that determines the ferromagnetism in graphene with vacancies via a never before reported mechanism, see the next subsection for details. From Table I, the MM for cases $5 \times 5 \sim 8 \times 8$ are $1.6 \mu_{\mathrm{B}}, 1.6 \mu_{\mathrm{B}}, 1.3 \mu_{\mathrm{B}}$ and $1.3 \mu_{\mathrm{B}}$, respectively. Except for the contribution of the $s p^{2 *}$ antibonding states, $1.0 \mu_{\mathrm{B}}$, the magnetic moment of 0.3 $\sim 0.6 \mu_{\mathrm{B}}$ can be traced to different electron occupations in the majority and minority of the $p_{z}^{*}$-nonbonding states. As discussed in the cases of $2 \times 2 \sim 4 \times 4$, this is a result of the competition between the kinetic energy and the exchange energy. For cases $7 \times 7$ and $8 \times 8$, more minority nonbonding states are occupied, reducing the MM.

\section{F. Unusual ferromagnetic mechanism distinctly different from the conventional mechanism}

As mentioned above, when the origin of the magnetism in proton-irradiated graphene was explained by the conventional magnetic theory, it was debated or resulted in conflicts. The current magnetic models, such as the indirect exchange model, the superexchange model, the double exchange model and the RKKY model, have the same scalar product in the exchange energy, $\mathbf{S}_{i} \cdot \mathbf{S}_{j}$; the difference lies only in the manner of coupling. In this aspect, these models are all based on Heisenberg's theory originated from the exchange integrals for an $\mathrm{H}_{2}$ molecule ${ }^{10}$. The magnetic mechanism for magnetic materials such as iron and nickel metals is governed by the conventional magnetic theory. 
If the conventional magnetic theory is used to explain the magnetism in graphene with vacancies, contradictions and difficulties lie at least in two aspects. 1) How can $s p$ electrons (principle quantum number $n<3$ ) be spin-polarized? 2) How can such a weak magnetization $\left(10^{-3} \sim 10^{-4}\right.$ orders of magnitude smaller than a conventional magnet) have such a high critical temperature? In other words, how is such a long-range (with a distance between vacancies of up to $20 \AA$ ) coupling strong enough to trigger ferromagnetic ordering above room temperature $e^{3|13| 29}$ ? There must be an unrecognized magnetic mechanism at work.

Concerning the difficulty in explaining $s p$ electron spin-polarization in proton-irradiated graphene, we have shown that the vacancy-induced states such as antibonding and nonbonding states can appear near the Fermi level. If these states are partially filled, according to the antisymmetric principle of electron exchange the $s p$ electrons could be spin-polarized. Obviously, unlike an isolated atom governed by Hund's rule, the $s p$-electron spin polarization in proton-irradiated graphene originates from the antisymmetric spatial wavefunctions involving three atoms surrounding the vacancy for $s p^{2 *}$ and all the atoms of the majority sublattice for $p_{z}^{*}$. The induced MM of the atoms are not individually localized but are distributing as a whole moment on all the involved atoms. If the entire magnetic moment is projected on the atoms, the projected moments appear to be distributed on the involved atoms. To distinguish these from localized MM, we refer to these moments as fractional magnetic moments. The fractional MM on the majority sublattice atoms, whose $p_{z}$ orbitals are composed in an antisymmetric manner, are inseparably combined into the entire magnetic moment, because they belong to one electronic state, the $p_{z}^{*}$ state. This is a distinctly different mechanism from any conventional mechanism. We used the word combine in the last sentence to emphasize that there is no coupling interaction between the fractional magnetic moments, rather the intrinsic parts belong to the entire magnetic moment. These fractional MM, as a whole moment induced by partially filled antibonding and nonbonding states, should be parallel according to the exchange antisymmetric principle for electrons.

Concerning the difficulty in explaining the ferromagnetic ordering with such a high critical temperature in proton-irradiated graphene, we have stressed in Subsection III A that we did not perform calculations for the exchange energy based on the conventional magnetic theory for two reasons. 1) Because the vacancy concentration in proton-irradiated graphene, for which ferromagnetism was observed, corresponds to a distance between vacancies as large as 15 20 A; a valid simulation would be computationally demanding. 2) Because such a longrange $(15 \sim 20 \AA)$ coupling itself is beyond any conventional magnetic models, it is not possible to obtain a significant conclusion from calculations based on the conventional magnetic theory.

In Subsection III we demonstrated that the nonbond- ing state, $p_{z}^{*}$, is not induced by an interaction between the atoms in the majority sublattice but by an imbalance between the $p_{z}$ orbitals of two sublattices due to the removal of one atom in the minority sublattice. We have shown via the TB model that, considering only the interaction between the first nearest neighbors $(1 \mathrm{NN}), p_{z}^{*}$ consists of the $p_{z}$-orbitals of only the atoms in the majority sublattice. Even when considering the interaction of the second nearest neighbors $(2 \mathrm{NN})$, the origin of the unpaired $p_{z}$-orbitals constrains the orbital components on the atoms of the minority sublattice as little as possible because any extra orbital components of $p_{z}^{*}$ on the minority sublattice would cause an extra imbalance between the two sublattices. Furthermore, as a point-defect state of a two-dimensional lattice resonating with energy of a perfect crystal, its wavefunction decays from the vacancy as $\sim 1 / r$, keeping the orbital components on the minority sublattice as little as possible within a region of the decay length. According to these characteristics, we conclude that $p_{z}^{*}$ plays a key role not only in spinpolarization but also in magnetic ordering, based on the following analysis.

Removing one atom from the minority sublattice causes one $p_{z}^{*}$ state and leaves one unpaired $p_{z}$ electron in the majority sublattice. Appearing near the Fermi level, the $p_{z}^{*}$ state will be filled by one remaining electron in spin polarization, leading to fractional MM distributed on the majority sublattice atoms. Removing two infinitely separated atoms in the minority sublattice could create two independent $p_{z}^{*}$ states and two free electrons.

Imaging that the two vacancies are moved closer and closer until the two regions of each $p_{z}^{*}$ within the decay length overlap, the two independent $p_{z}^{*}$ states are therefore coherent. Within the overlap region, this favors keeping the wavefunction components as little as possible on the atoms of the minority sublattice because the $p_{z}^{*}$ states themselves do not originate from an interaction, but from an imbalance between the $p_{z}$-orbitals of the two sublattices, corresponding to a recombination of the unpaired $p_{z}$-orbitals of the majority sublattice atoms. The unpaired nature again constrains the sequence antisymmetric wavefunction extending from the overlap region to all the involved regions, leading to the two $p_{z}^{*}$ being coherent if they are within the decay length.

As mentioned above, in addition to the $p_{z}^{*}$ in graphene with a vacancy, the antibonding state, $s p^{2 *}$, which is short ranged, induces fractional magnetic moments on the three atoms surrounding the vacancy. Here $p_{z}^{*}$ can be seen as acting as an effective magnetic field to polarize the spin of the electrons filling on the $s p^{2 *}$ state because both $p_{z}^{*}$ and $s p^{2 *}$ originate all from the same vacancy.

The above analysis, in two respects (spin-polarization and magnetic ordering), indicates a never before reported mechanism that is different from the conventional mechanism. Different models for magnetic ordering, such as the direct exchange model, the indirect exchange model, the superexchange model, the double exchange model and the RKKY model can be all traced to the same mech- 
anism with different coupling manners. However, there are no localized MM in this new unusual magnetic mechanism, instead, there are fractional MM of the entire moment that are always aligned parallel due to the antisymmetric wavefunctions. Note that the coherent origin of $p_{z}^{*}$ is not an interaction between vacancies but the imbalance between the $p_{z}$ orbitals of the two sublattices; as in the origin of an isolated $p_{z}^{*}$ state, recombining the unpaired $p_{z}$-orbitals caused by more vacancies keeps the wavefunction of $p_{z}^{*}$ as little as possible in the minority sublattice.

The temperature plays a role in the magnetic ordering for this unusual mechanism: while it does not act as a factor to decouple the MM, but it does destroy the nonbonding state itself or does change the electron filling situation, that is, the involved state is shifted from being partially to fully filled or from being partially filled to completely empty. This is why such a weak magnetization can be bewilderingly observed at room temperature in proton-irradiated graphene.

In this way, we can also explain the existence of a small window for vacancy concentration. A previous study concluded that, if vacancy concentration is too large, localized $s p$-electrons become delocalized and therefore destroy the FM ordering; therefore, there is a small window of vacancy concentration, up to which no ferromagnetism can be observed ${ }^{8}$. We have stressed that no really localized electrons exist in band calculations based on SEA. According to the above analysis, we propose an alternative possibility that large vacancy concentrations mean that more electrons are released and more $p_{z}^{*}$ states are formed. The $p_{z}^{*}$ states, saying two per unit cell, can make contact with each other to form two combined states with some energy splitting. (if considering also $2 \mathrm{NN}$ interaction in TB model). If the energy splitting of the two combined states is larger than the spin splitting, the two electrons released by two vacancies will fully fill the lower combined state without spin polarization, therefore destroying the ferromagnetism.

\section{CONCLUSIONS}

Based on first principle calculations, we traced for the first time the magnetism in nominally nonmagnetic materials to the antisymmetric orbital combination of the involved electronic states and proposed a never before reported magnetic mechanism distinctly different from the conventional theory. We emphasize that in these two respects, our conclusions are different from the previous investigations.

We investigated the origin of magnetism in graphene with vacancies. It was shown that MM existed in all the investigated cases (for $2 \times 2 \sim 8 \times 8$ ), varying between $1.0 \mu_{\mathrm{B}}$ and $1.6 \mu_{\mathrm{B}}$ and reaching a stable value of $1.3 \mu_{\mathrm{B}}$ in the $7 \times 7$-sized cell. The compelling evidence shows that the MM in materials with only $s p$ electrons can be traced to the antisymmetric manner of the wavefunctions of the involved electronic states, the $s p^{2 *}$-antibonding and $p_{z}^{*}$-nonbonding states which appear near the Fermi level. This is a conclusion distinctly different from the previous investigations as pointed out in section Introduction. Removing one atom from graphene creates a vacancy, leaving three dangling bonds on three atoms pointing toward the vacancy and breaking the balance between the $p_{z}$ orbitals of the two sublattices. Consequently, the three $s p^{2}$ dangling bonds interact with each other to hybridize one bonding state and two antibonding states without JTD (for $2 \times 2$ and $3 \times 3$ ) or with JTD (for $4 \times 4 \sim 8 \times 8$ ); in addition, the unpaired $p_{z}$ orbital on the majority sublattice atoms forms one nonbonding state. Because the spatial wavefunctions of both the $s p^{2 *}$-antibonding and $p_{z}^{*}$-nonbonding states are antisymmetric, their spin wavefunctions should be symmetric according to the electron exchange antisymmetric principle. Appearing near the Fermi level, $s p^{2 *}$ and $p_{z}^{*}$ will be partially filled in spin polarization. This is the origin of $s p$-electron spin-polarization in proton-irradiated graphene. We emphasize that, unlike the conventional magnetic models, the $p_{z}^{*}$ induced MM are fractional MM of a whole moment distributed not on one atom but on all the involved atoms.

The nonbonding state stems not from an interaction between atoms but from an imbalance between the $p_{z}$ orbitals of the two sublattices due to removing one atom from the minority sublattice. Therefore, in addition to contributing fractional MM, the nonbonding state plays a critical role in magnetic ordering. If the vacancy concentration is large enough to cause the vacancy-affected regions to overlap each other, the requirement of as little orbital components as possible on the minority sublattice in the overlap regions makes the vacancy-induced $p_{z}^{*}$ states coherent because more vacancies mean more unpaired $p_{z}$-orbitals, which require recombination. The coherent process in the overlap region therefore constrains the antisymmetric wavefunction covering all the vacancy-affected regions, consequently causing ferromagnetism according to the electron exchange antisymmetric principle. Therefore, we can understand how, in proton-irradiated graphene, such far-flung spins can be so strongly aligned and cannot be destroyed even by a high temperature $(>300 \mathrm{~K})$. Obviously, the connecting thread is a mechanism that is different from any previously published models for magnetic ordering.

This work was supported by NFSC (No.61274097) and NBRPC (No. 2015CB921401).

\footnotetext{
* Corresponding author. E-mail: jgche@fudan.edu.cn.
}

1 T. Makarova and F. Palacio, eds., Carbon Based Mag- 
netism: An Overview of the Magnetism of Metal Free Carbon-Based Compounds and Materials (Elsevier, Amsterdam, 2006).

2 O. V. Yazyev, Reports on Progress in Physics 73, 056501 (2010).

3 P. Esquinazi, W. Hergert, D. Spemann, A.Setzer, and A. Ernst, IEEE Transactions on Magnetics 49, 4668 (2013).

${ }^{4}$ Y. P. Feng, L. Shen, M. Yang, A. Wang, M. Zeng, Q. Wu, S. Chintalapati, and C.-R. Chang, WIREs Computational Molecular Science 7, e1313 (2017).

5 B. Náfrádi, M. Choucair, and L. Forró, Advanced Functional Materials 27, 1604040 (2017).

6 A. L. Kuzemsky, International Journal of Modern Physics B 27, 1330007 (2013).

7 W. Han, R. K. Kawakami, M. Gmitra, and J. Fabian, Nature Nanotechnology 9, 794 (2014).

8 P. Fischer and H. Ohldag, Reports on Progress in Physics 78, 094501 (2015).

9 R. R. Nair et al., Nature Physics 8, 199 (2012).

10 W. Heisenberg, Zeitschrift für Physik 49, 619 (1928).

11 N. García, in Carbon Based Magnetism: An Overview of the Magnetism of Metal Free Carbon-Based Compounds and Materials, edited by T. Makarova and F. Palacio (Elsevier, Amsterdam, 2006) pp. ix - xi.

12 P. Esquinazi, A. Setzer, R. Höhne, C. Semmelhack, Y. Kopelevich, D. Spemann, T. Butz, B. Kohlstrunk, and M. Lösche, Phys. Rev. B 66, 024429 (2002).

13 M. M. Ugeda, I. Brihuega, F. Guinea, and J. M. GómezRodríguez, Phys. Rev. Lett. 104, 096804 (2010).

14 J. M. D. Coey, M. Venkatesan, C. B. Fitzgerald, A. P. Douvalis, and I. S. Sanders, Nature 420, 156 (2002).

15 M. I. Katsnelson, Graphene: Carbon in Two Dimensions (Cambridge University Press, Cambridge, 2012).

16 R. Singh, Journal of Magnetism and Magnetic Materials 346, 58 (2013).

17 P. Esquinazi, D. Spemann, R. Höhne, A. Setzer, K.-H. Han, and T. Butz, Phys. Rev. Lett. 91, 227201 (2003).

18 J. Červenka, M. I. Katsnelson, and C. F. J. Flipse, Nature Physics 5, 840 (2009).

19 Y. Wang, Y. Huang, Y. Song, X. Zhang, Y. Ma, J. Liang, and Y. Chen, Nano Letters 9, 220 (2009).

${ }^{20}$ P. O. Lehtinen, A. S. Foster, A. Ayuela, A. Krasheninnikov, K. Nordlund, and R. M. Nieminen, Phys. Rev. Lett. 91, 017202 (2003).

21 P. O. Lehtinen, A. S. Foster, Y. Ma, A. V. Krasheninnikov, and R. M. Nieminen, Phys. Rev. Lett. 93, 187202 (2004).

${ }^{22}$ V. M. Pereira, F. Guinea, J. M. B. Lopes dos Santos, N. M. R. Peres, and A. H. Castro Neto, Phys. Rev. Lett. 96, 036801 (2006).

23 O. V. Yazyev and L. Helm, Phys. Rev. B 75, 125408 (2007).

24 J. J. Palacios, J. Fernández-Rossier, and L. Brey, Phys. Rev. B 77, 195428 (2008).

25 S. Lisenkov, A. N. Andriotis, and M. Menon, Phys. Rev. B 82, 165454 (2010).

26 J. J. Palacios, and F. Ynduráin, Phys. Rev. B 85, 245443 (2012).
27 B. R. K. Nanda, M. Sherafati, Z. S. Popović, and S. Satpathy, New Journal of Physics 14, 083004 (2012).

28 C. Kittel, Introduction to Solid State Physics, 8th ed. (Wiley, New York, 2004).

29 I. S. Elfimov, S. Yunoki, and G. A. Sawatzky, Phys. Rev. Lett. 89, 216403 (2002).

${ }^{30}$ V. M. Pereira, J. M. B. Lopes dos Santos, and A. H. Castro Neto, Phys. Rev. B 77, 115109 (2008).

31 Y. Kopelevich, P. Esquinazi, J. H. S. Torres, and S. Moehlecke, Journal of Low Temperature Physics 119, 691 (2000).

32 L. Pisani, B. Montanari, and N. M. Harrison, New Journal of Physics 10, 033002 (2008).

33 M. Sepioni, R. R. Nair, S. Rablen, J. Narayanan, F. Tuna, R. Winpenny, A. K. Geim, and I. V. Grigorieva, Phys. Rev. Lett. 105, 207205 (2010).

34 H. González-Herrero, J. M. Gómez-Rodríguez, P. Mallet, M. Moaied, J. J. Palacios, C. Salgado, M. M. Ugeda, J.-Y. Veuillen, F. Yndurain, and I. Brihuega, Science 352, 437 (2016).

35 G. Kresse and J. Furthmüller, Computational Materials Science 6, 15 (1996); G. Kresse and J. Furthmüller, Phys. Rev. B 54, 11169 (1996); G. Kresse and D. Joubert, Phys. Rev. B 59, 1758 (1999).

36 P. E. Blöchl, Phys. Rev. B 50, 17953 (1994).

37 J. P. Perdew, K. Burke, and M. Ernzerhof, Phys. Rev. Lett. 77, 3865 (1996).

38 J. P. Perdew and A. Zunger, Phys. Rev. B 23, 5048 (1981).

39 A. H. Castro Neto, F. Guinea, N. M. R. Peres, K. S. Novoselov, and A. K. Geim, Rev. Mod. Phys. 81, 109 (2009).

40 A. A. Mostofi, J. R. Yates, Y.-S. Lee, I. Souza, D. Vanderbilt, and N. Marzari, Computer Physics Communications 178, 685 (2008); N. Marzari and D. Vanderbilt, Phys. Rev. B 56, 12847 (1997); I. Souza, N. Marzari, and D. Vanderbilt, Phys. Rev. B 65, 035109 (2001).

41 Y. Ma, P. O. Lehtinen, A. S. Foster, and R. M. Nieminen, New Journal of Physics 6, 68 (2004).

${ }^{42}$ H. Padmanabhan and B. R. K. Nanda, Phys. Rev. B 93, 165403 (2016).

43 P. Vollhardt and N. Schore, Organic Chemistry: Structure and Function, 6th ed. (W. H. Freeman and Company, New York, 2011).

44 D. J. Griffiths, Introduction to Quantum Mechanics, 2nd ed. (Cambridge University Press, Cambridge, 2016).

45 J.-X. Yu and J. G. Che, Phys. Rev. B 93, 035449 (2016).

46 J.-X. Yu and J. G. Che, Europhysics Letters 113, 17008 (2016).

$47 \mathrm{~W}$. Xu, J. Shang, J. X. Yu, and J. G. Che, arXiv: 1808.03505

48 E. J. G. Santos, A. Ayuela, and D. Sánchez-Portal, New Journal of Physics 12, 053012 (2010).

49 M. Sun, Q. Ren, Y. Zhao, J.-P. Chou, J. Yu, and W. Tang, Carbon 120, 265 (2017).

50 J. Dabrowski and H.-J. Müssig, Silicon Surfaces and Formation of Interfaces (World Scientific, Singapore, 2000). 